\title{
Enthesopathy and tendinopathy in gout: computed tomographic assessment
}

\author{
Jean-Charles Gerster, Michel Landry, Georges Rappoport, Gilles Rivier, Bertrand \\ Duvoisin, Pierre Schnyder
}

\begin{abstract}
Objective-To establish if computed tomography (CT) imaging, which has proved helpful in detecting intra-articular tophi in gout, can also be used to document gouty enthesopathy and tendinopathy.

Methods-Three patients with tophaceous gout and clinical involvement of the Achilles tendon (two cases) or patellar tendon (one case) were assessed with CT examination and plain radiographs.

Results-In the first two cases, CT images revealed linear or nodular high attenuation opacities within the substance of the Achilles tendons and their calcaneal insertion. In case 3, dense linear opacities were seen within the patellar tendon and within its tibial insertion. No such opacities of the tendons and entheses were seen on standard radiographs of these patients.

Conclusions-CT appears to be the imaging method of choice for demonstrating monosodium urate deposits in entheses and tendons in tophaceous gout.
\end{abstract}

(Ann Rheum Dis 1996;55:921-923)

Tendinopathy, particularly of the Achilles and patellar tendons, is a fairly common presentation of gout and can even lead to their rupture. ${ }^{1}$ Clinical involvement of these tendons can precede any articular gouty manifestation, as found in 12 out of 17 cases of tendinopathy of the Achilles tendon. ${ }^{2}$ More recently, gouty enthesopathy-mainly of the heels-has been reported in some renal transplant recipients who were receiving cyclosporin treatment, despite the absence of microcrystalline joint effusion. ${ }^{3}$ Imaging of the gouty Achilles tendon with plain radiographs or xeroradiographs reveals a diffuse or nodular thickening that cannot be distinguished from xanthomata or nodular thickening due to partial rupture. ${ }^{4}$ We have previously shown that computed tomography (CT) was useful in documenting tophaceous deposits of monosodium urate crystals within the knee joint: the tophi are of greater attenuation than the muscles, and their density is 170 (SD 15) Hounsfield Units (HU). ${ }^{5}$ In this study of three patients with tophaceous gout, the ability of CT to identify monosodium urate deposits in clinically involved tendons (Achilles tendon in two patients, patellar tendon in one patient) was assessed.

\section{Case reports}

PATIENT 1

A 70 year old man was admitted with acute arthritis of the left ankle joint. He had been suffering from gout for 10 years, and had a history of excessive alcohol consumption and of irregular medication consisting of nonsteroidal anti-inflammatory drugs and allopurinol.

Upon admission, the patient was overweight (body mass index $32.8 \mathrm{~kg} \mathrm{~m}^{-2}$, normal < 25), and he had an effusion of the left knee, signs of acute arthritis of the left ankle, and nodules of both Achilles tendons, which were slightly tender on palpation; he also had nodules next to the olecranon processes. The arteries of both feet were pulsatile; blood pressure was $170 / 80$ $\mathrm{mm} \mathrm{Hg}$. Synovial fluid aspirated from the left knee contained 1000 leucocytes $\mathrm{mm}^{-3}$ with intracellular negatively birefringent crystals. Serum uric acid was $455 \mu \mathrm{mol}$ litre $^{-1}$ (normal < 416); creatinine $80 \mu \mathrm{mol}$ litre ${ }^{-1}$ (normal < 106); fasting blood sugar $7.0 \mathrm{mmol}$ litre $^{-1}$; and cholesterol $4.2 \mathrm{mmol} \mathrm{litre}^{-1}$ (normal < 5.2). Plain radiographs of both knees did not reveal chondrocalcinosis. The lateral radiographs of both ankles were normal except for calcifications of the posterior tibial arteries (fig 1A). A CT scan was achieved with a $9800 \mathrm{High}$ Speed Advantage GE scanner, using $120 \mathrm{KV}$, $140 \mathrm{mAs}, 1 \mathrm{~mm}$ collimation (table speed 1 $\mathrm{mm} / \mathrm{s}$, pitch 1). The CT sections revealed opacities in the posterior part of the Achilles tendon, undetected on the plain radiographs (fig $1 \mathrm{~B}$ ), and were considered to be monosodium urate deposits based on their density, that is, 170 (15) HU.

PATIENT 2

A man aged 42 years who had had gout for 15 years presented articular pains of both elbows and right knee and inflammation of the posterior aspect of both feet. There was a history of chronic excessive alcohol consumption and of hyperuricaemia treated irregularly.

The patient was obese (body mass index 33 $\mathrm{kg} \mathrm{m}^{-2}$, and upon examination an effusion of the knee joints, nodular thickening of both 

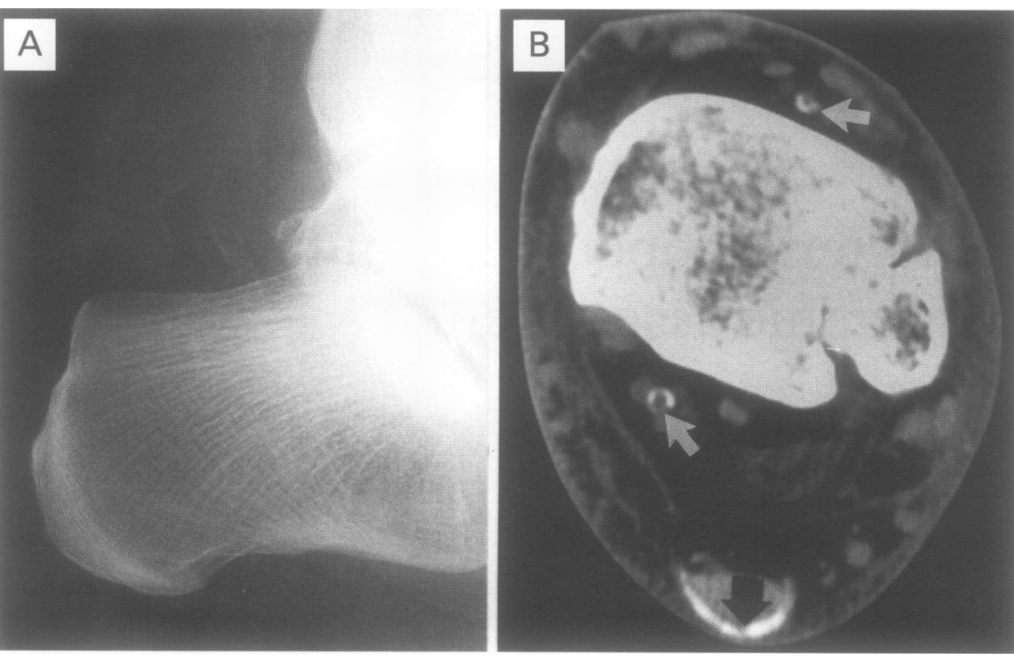

Figure $1(A)$ Lateral radiograph of the left heel (case 1) showing vascular calcifications. The Achilles tendon is slightly enlarged. (B) Same heel. CT axial image; vascular calcifications are seen (white arrows) as well as opacities of the posterior part of the Achilles tendon [mean density 170 (SD 15) HU], considered to represent monosodium urate crystal deposits (black arrow). tendon. In 1993, an arthroscopic examination of the knee revealed a thickened synovium and white crystal deposits on the synovium and in the internal meniscus. There was also a history of chronic alcohol abuse and poor observance of the antihyperuricaemic treatment.

In 1995, the patient was overweight (body mass index $32.9 \mathrm{~kg} \mathrm{~m}^{-2}$ ). Upon examination, he had tophi of the right wrist and of the second left finger joint. The left knee was swollen with an enlarged prepatellar bursa and the patellar tendon diffusely swollen and tender at its tibial insertion. Laboratory results showed birefringent negative monosodium urate crystals in a paucicellular synovial fluid aspirated from the left knee joint. Serum uric acid was $577 \mu \mathrm{mol}$ litre $^{-1}$; creatinine $109 \mu \mathrm{mol}$ litre $^{-1}$; fasting glucose $12.4 \mathrm{mmol} \mathrm{litre}^{-1}$; and cholesterol 6.4 mmol litre ${ }^{-1}$.

Anteroposterior radiographs of the left knee joint revealed discrete signs of osteoarthritis but no chondrocalcinosis. On the lateral view, there were no opacities of the soft tissues (fig $3 \mathrm{~A})$. In contrast CT imaging showed irregular

Achilles tendons, and a tender and swollen calcaneal insertion were noted. The blood pressure was $120 / 90 \mathrm{~mm} \mathrm{Hg}$. Laboratory results revealed negatively birefringent crystals in a paucicellular synovial fluid aspirated from the right knee joint; serum uric acid was 609 $\mu \mathrm{mol}$ litre $^{-1}$; creatinine $76 \mu \mathrm{mol}$ litre $^{-1}$; glucose $5.5 \mathrm{mmol}^{\text {litre }}{ }^{-1}$.

Lateral radiographs of the heels did not reveal opacities of the soft tissues, but CT imaging showed opacities within the Achilles tendon, in the subcutaneous tissue, and at the calcaneal insertion, clearly demonstrated on a sagittal cross sectional reconstruction (fig 2).

PATIENT 3

A 69 year old man with gout of 20 years' duration had some years previously undergone an excision of the right olecranon bursa for chronic gouty bursitis as well as a decompression of the right carpal tunnel. At that time, macroscopic tophaceous deposits were observed in the flexor tendons. He suffered occasionally from inflammatory bouts of the left prepatellar bursa and patellar

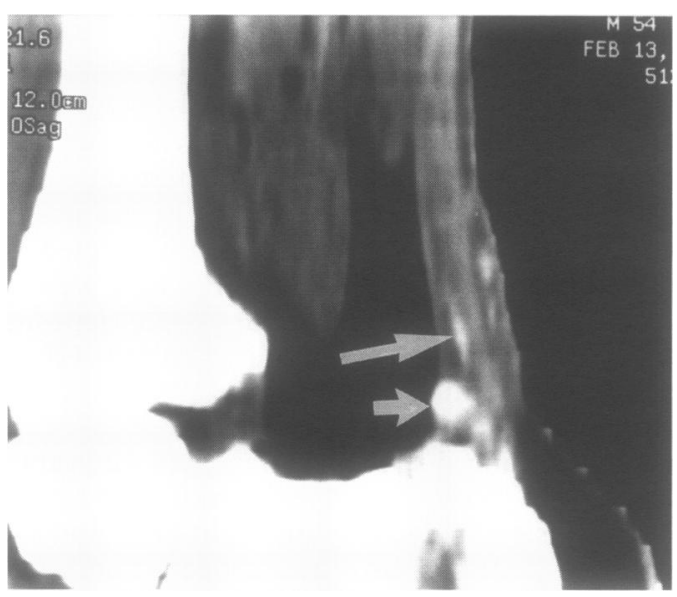

Figure 2 Computerised tomographic cross sectional sagittal image reconstruction of the right heel (case 2). The Achilles tendon is thickened. There are linear opacities within the tendon (large arrow), in the subcutaneous tissue and at the calcaneal insertion (preachilles bursa) (small arrow) [mean density 170 (SD 15) HU].
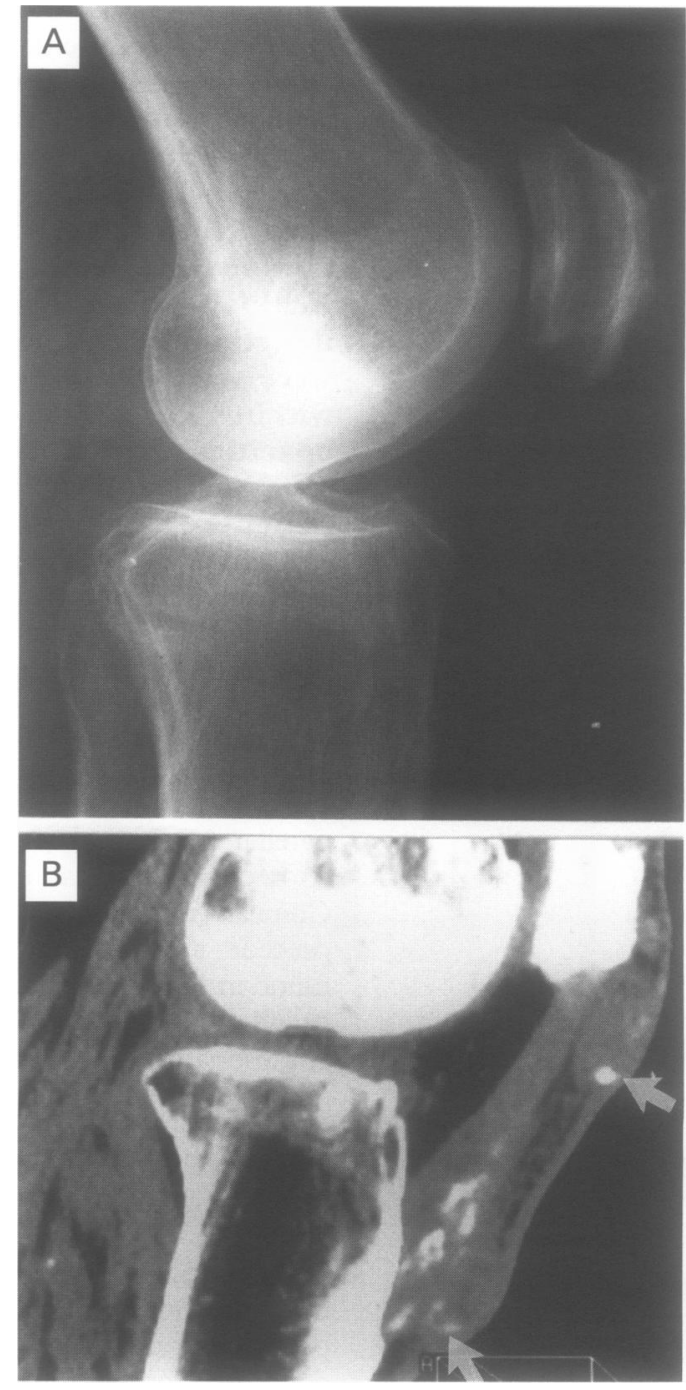

Figure 3 (A) Lateral radiograph of the left knee (case 3). $A$ periosteal reaction along the anterior part of the patella is seen. The patellar tendon is slightly enlarged. No opacity is identified. (B) Computerised tomographic cross sectional sagittal image reconstruction (same knee) showing opacities within the tendon and in front of its tibial insertion. Two subcutaneous bursae are seen (arrows) and contain round opacities. 
round and oval opaque masses within the prepatellar bursa, the patellar tendon, and at its tibial insertion, clearly visible on a sagittal cross sectional reconstruction (fig $3 \mathrm{~B}$ ).

\section{Discussion}

Symptomatic Achilles tendon and patellar tendon were selected for this study. These tendons are commonly involved in gout ${ }^{26}$; since they are devoid of a synovial sheath, synovial fluid cannot be obtained and the documentation of the microcrystalline pathology is not easy. However, as we have shown previously, ${ }^{5} \mathrm{CT}$ techniques reveal monosodium urate deposits in vitro as well as within the knee joint, while such deposits are not visible on plain radiographs. ${ }^{5}$ Increased attenuation of the $x$ ray beam of the CT scanner, as reflected by an increased $\mathrm{HU}$ value [170 (SD 15) HU], could be due to a high concentration of sodium nuclei in the monosodium urate crystals. It is well known that CT scanning can readily diagnose stones of the urinary tract not visible on conventional radiographs ${ }^{7}$; it can be assumed that such calculi are composed mainly of urate. Although in our cases no pathological correlation was obtained, tophaceous deposits within the Achilles tendon have been the subject of histological investigations by others ${ }^{12}$ and are clearly documented.

CT examination of the tendons could permit differentiation of tendinous tophi from hypercholesterolaemic xanthomata: there is no increased density of the latter and their $x$ ray attenuation is similar to that of muscles. ${ }^{8}$

Our study shows that monosodium urate deposits can be found at insertions of the tendons and in the surrounding structure, causing gouty enthesopathy. This is very similar to the enthesopathy described in CPPD crystal deposition disease.' In this latter condition, however, CPPD crystal deposits are clearly apparent on radiographs and xeroradiographs as fine linear calcifications.' Monosodium urate deposits at the entheses, which are not visible on conventional radiographs, have also to be distinguished from numular apatite deposits, distinctly seen on radiographs, ${ }^{10}$ and finally from ossifications near the insertion of the Achilles tendon which may occur in diffuse idiopathic skeletal hyperostosis (DISH), ${ }^{11}$ well shown on conventional $x$ ray films.

CT should not be done routinely but it could become a method of choice for assessing certain cases of tendinous thickening of possibly gouty origin. It can characterise the crystal deposits by their shape and their density, providing different information from magnetic resonance imaging, which has recently been shown to have a place in the evaluation of tophaceous gout of the hands. ${ }^{12}$

Long term studies are needed to determine if CT could be helpful in monitoring antihyperuricaemic treatment and whether the abnormalities are reversible.

1 Mahoney PG, James PD, Howell CJ, Swannell AJ. Spontaneous rupture of the Achilles tendon in a patient with gout. Ann Rheum Dis 1981;40:416-8.

2 Commandre F, Viani JL, Hammou JC, Revelli G. A propos de 28 cas de tendinopathies goutteuses. Rhumatologie 1978;30:2738.

3 Cohen MR, Cohen EP. Enthesopathy and atypical gouty arthritis following renal transplantation: a case control study. Rev Rhum (English ed) 1995; 62:86-90.

4 Gerster JC, Hauser H, Fallet GH. Xeroradiographic techniques applied to assessment of Achilles tendon in inflammatory or metabolic diseases. Ann Rheum Dis 1975; 34:479-88.

5 Gerster JC, Landry M, Duvoisin B, Rappoport G. Computed tomography of the knee joint as an indicator of
intraarticular tophi in gout. Arthritis Rheum 1996; 39:1406-9.

6 Dieppe P, Calvert P. Gout. In: Dieppe P, Calvert P, eds. Crystals and joint disease. London: Chapman \& Hall, 1983:115-53.

7 Dean TE, Harrison NW, Bischop NL. CT scanning in the diagnosis and management of radiolucent urinary calculi. Br $\mathcal{f}$ Urol 1988;62:405-8.

8 Hertzanu Y, Berginer J, Berginer VM. Computed tomography of tendinous xanthoma in cerebrotendinous xanthomatosis. Skel Radiol 1991;20:99-102.

9 Gerster JC, Baud CA, Lagier R, Boussina I, Fallet GH. Tendons calcifications in chondrocalcinosis. A clinical, radiologic, histologic and crystallographic study. Arthritis Rheum 1977;20:717-22.

10 Resnick D, Niwayama G. Entheses and enthesopathy. Anatomical, pathological and radiological correlation. Radiology 1983;146:1-9.

11 Gerster JC. Achilles tendinitis as a severe clinical feature of diffuse idiopathic skeletal hyperostosis. $\mathcal{f}$ Rheumatol 1995; 22:1212-3.

12 Popp JD, Bidgood WD, Edwards NL. Magnetic resonance imaging of tophaceous gout in the hands and wrists. Semin Arthritis Rheum 1996;25:282-9. 